\title{
Latissimus Dorsi and Teres Major Transfer With Reverse Shoulder Arthroplasty Restores Active Motion and Reduces Pain for Posterosuperior Cuff Dysfunction
}

\author{
Lewis L. Shi MD, Kirk E. Cahill BA, Eugene T. Ek MBBS, PhD, Jeffrey D. Tompson MS, \\ Laurence D. Higgins MD, Jon J. P. Warner MD
}

Received: 10 January 2015/ Accepted: 25 June 2015/Published online: 3 July 2015

(C) The Association of Bone and Joint Surgeons (B) 2015

\begin{abstract}
Background In patients with rotator cuff dysfunction, reverse shoulder arthroplasty can restore active forward flexion, but it does not provide a solution for the lack of active external rotation because of infraspinatus and the teres minor dysfunction. A modified L'Episcopo procedure can be performed in the same setting wherein the latissimus dorsi and teres major tendons are transferred to the lateral aspect of proximal humerus in an attempt to restore active external rotation.
\end{abstract}

Each author certifies that he or she, or a member of his or her immediate family, has no funding or commercial associations (eg, consultancies, stock ownership, equity interest, patent/licensing arrangements, etc) that might pose a conflict of interest in connection with the submitted article.

All ICMJE Conflict of Interest Forms for authors and Clinical Orthopaedics and Related Research ${ }^{\mathbb{B}}$ editors and board members are on file with the publication and can be viewed on request.

Clinical Orthopaedics and Related Research ${ }^{\mathbb{R}}$ neither advocates nor endorses the use of any treatment, drug, or device. Readers are encouraged to always seek additional information, including FDAapproval status, of any drug or device prior to clinical use.

Each author certifies that his or her institution approved the human protocol for this investigation, that all investigations were conducted in conformity with ethical principles of research, and that informed consent for participation in the study was obtained

This work was performed at Massachusetts General Hospital and Brigham and Women's Hospital, Boston, MA, USA.

L. L. Shi, K. E. Cahill

Department of Orthopaedic Surgery and Rehabilitation

Medicine, The University of Chicago Hospitals, Chicago, IL, USA

\section{E. T. Ek}

Melbourne Orthopaedic Group and the Department of Orthopaedic Surgery, Austin Hospital, University of Melbourne,

Melbourne, Australia
Questions/purposes (1) Do latissimus dorsi and teres major tendon transfers with reverse shoulder arthroplasty improve external rotation function in patients with posterosuperior rotator cuff dysfunction? (2) Do patients experience less pain and have improved outcome scores after surgery? (3) What are the complications associated with reverse shoulder arthroplasty with latissimus dorsi and teres major transfer?

Methods Between 2007 and 2010, we treated all patients undergoing shoulder arthroplasty who had a profound external rotation lag sign and advanced fatty degeneration of the posterosuperior rotator cuff (infraspinatus plus teres minor) with this approach. A total of 21 patients (mean age 66 years; range, 58-82 years) were treated this way and followed for a minimum of 2 years (range, 26-81 months); none was lost to followup, and all have been seen in the last 5 years. We compared pre- and postoperative ranges of motion, pain, and functional status; scores were drawn from chart review. We also categorized major and minor complications.

Results Active forward flexion improved from $56^{\circ} \pm 36^{\circ}$ to $120^{\circ} \pm 38^{\circ}$ (mean difference: $64^{\circ}$ [95\% confidence interval $\left.\left.\{\mathrm{CI}\}, 45^{\circ}-83^{\circ}\right], \mathrm{p}<0.001\right)$. Active external rotation with the arm adducted improved from $6^{\circ} \pm 16^{\circ}$ to $38^{\circ}$ $\pm 14^{\circ}$ (mean difference: $30^{\circ}$ [95\% CI, $21^{\circ}-39^{\circ}$ ], p < 0.001 ); active external rotation with the arm abducted improved from $19^{\circ} \pm 25^{\circ}$ to $74^{\circ} \pm 22^{\circ}$ (mean difference:

\footnotetext{
J. D. Tompson, J. J. P. Warner ( $\square)$

Harvard Shoulder Service, Massachusetts General Hospital,

55 Fruit Street, Boston, MA 02114, USA

e-mail: jwarner@partners.org

L. D. Higgins

Sports Medicine and the Shoulder Service, Brigham and

Women's Hospital, Boston, MA, USA
} 
$\left.44^{\circ}\left[95 \% \mathrm{CI}, 22^{\circ}-65^{\circ}\right], \mathrm{p}<0.001\right)$. Pain visual analog score improved from $8.4 \pm 2.3$ to $1.7 \pm 2.1$ (mean difference: -6.9 [95\% CI, -8.7 to -5.2$], \mathrm{p}<0.001$ ), and Single Assessment Numeric Evaluation score improved from $28 \% \pm 21 \%$ to $80 \% \pm 24 \%$ (mean difference: $46 \%$ [95\% CI, 28\%-64\%], p < 0.001). There were six major complications, five of which were treated operatively. Overall, three patients' latissimus and teres major transfer failed based on persistent lack of external rotation.

Conclusions In patients with posterior and superior cuff deficiency, reverse shoulder arthroplasty combined with latissimus dorsi and teres major transfer through a single deltopectoral incision can reliably increase active forward flexion and external rotation. Patients experience pain relief and functional improvement but have a high rate of complications; therefore, we recommend the procedure be limited to patients indicated for reverse who have profound external rotation loss and a high grade of infraspinatus/teres minor fatty atrophy. Level of Evidence Level IV, therapeutic study.

\section{Introduction}

In patients with rotator cuff deficiency, reverse shoulder arthroplasty can restore active forward flexion; however, improving active external rotation is limited in the presence of dysfunctional infraspinatus and teres minor $[4,7,12,13]$. If posterior cuff deficiency is not attended to, the lack of active external rotation can compromise the outcome of the reverse arthroplasty [3, 11, 12]. A modified L'Episcopo technique where latissimus dorsi and teres major transfers are combined with reverse shoulder arthroplasty through a single deltopectoral approach has been described for patients with combined loss of active flexion and external rotation [1,8].

There is limited evidence in the literature for the treatment of this pathology with several small case series with short followup. These case series do not have clearly defined and uniform surgical indications and use different techniques that involve one versus two incisions and different tendon fixation methods.

We therefore asked: (1) Do latissimus dorsi and teres major tendon transfers with reverse shoulder arthroplasty improve external rotation function? (2) Do patients experience less pain and have improved outcome scores after surgery? (3) What are the complications associated with reverse shoulder arthroplasty with latissimus dorsi/teres major transfer?

\section{Patients and Methods}

Between February 2007 and July 2010, a total of 21 patients (mean age 66 years; range, 58-82 years) were treated with reverse shoulder arthroplasty with latissimus dorsi and teres major transfer as a single procedure and followed for a minimum of 2 years (range, 26-81 months); none was lost to followup, and all have been seen in the last 5 years (Table 1 ).

All patients demonstrated combined loss of active shoulder elevation and external rotation. Beyond the typical indication for reverse arthroplasty, the indications for the additional latissimus and teres major transfer included external rotation lag of more than $30^{\circ}$ with the arm in either adduction or abduction. These patients all exhibited atrophy/fatty infiltration of infraspinatus (Goutallier 3 or 4 ) and teres minor (Goutallier 2, 3, or 4). All patients in the study period meeting the surgical indication underwent this procedure. All patients were unable to maintain the affected arm in neutral position and all patients had a functional deltoid muscle on clinical examination. Fifteen of 21 patients had cuff tear arthropathy based on examination and preoperative imaging, and six patients had a proximal humerus fracture malunion/nonunion based on radiographs and CT scans. In seven of 21 patients (33\%), reverse shoulder arthroplasty with latissimus dorsi and teres major transfer was the primary procedure. The remaining 14 patients averaged 1.4 procedures before this with one of these being hemiarthroplasty. The group of patients with previous surgery was assessed with white blood cell count and C-reactive protein levels, and infection was excluded in all patients.

The surgical technique is similar to that originally described by Boileau and colleagues [2]. The reverse shoulder arthroplasty and the latissimus dorsi and teres major transfer were performed through a single deltopectoral approach (Fig. 1). Two surgeons performed all the procedures, using a similar technique, and using the Zimmer Anatomical Inverse system (Zimmer, Warsaw, IN, USA). Cementless fixation of the humerus stem was performed in the 15 patients undergoing cuff tear arthropathy, and cemented fixation of the humerus stem was used in the six patients with fracture. Tendon transfers were secured with multiple transosseous sutures on the lateral aspect of the humerus at the level of native latissimus and teres major insertion.

The patient's arm was placed in a neutral rotation brace for 6 weeks with no active ROM. At Week 4, physical therapy was initiated with passive elevation and external rotation $\left(30^{\circ}-40^{\circ}\right)$ but no internal rotation. From Weeks 6 to 12 , active assisted ROM was begun, leading to active ROM. During Weeks 10 to 12 , there was also biofeedback training consisting of a portable unit that provides audible and visual feedback to the patient about latissimus dorsi contraction during isometric shoulder adduction. In the subsequent phase, the patient coupled isometric shoulder adduction with external rotation to facilitate the activation of the transferred tendons. 
Table 1. Patient cohort and their characteristics

\begin{tabular}{|c|c|c|c|c|c|}
\hline Patient number & Sex & Age at operation (years) & Diagnosis & Followup (months) & Prior surgeries \\
\hline 1 & M & 67 & CTA & 81 & 3 (ORCR) \\
\hline 2 & $\mathrm{~F}$ & 77 & CTA & 65 & 0 \\
\hline 3 & $\mathrm{~F}$ & 68 & CTA & 45 & 2 (ARCR) \\
\hline 4 & $\mathrm{~F}$ & 58 & PHF (nonunion) & 63 & 1 (ORIF) \\
\hline 5 & $\mathrm{~F}$ & 63 & CTA & 52 & 0 \\
\hline 6 & $\mathrm{~F}$ & 63 & CTA & 50 & 0 \\
\hline 7 & $\mathrm{~F}$ & 68 & PHF (malunion) & 51 & 1 (ORIF) \\
\hline 8 & $\mathrm{~F}$ & 59 & CTA & 62 & 1 (ORCR) \\
\hline 9 & M & 80 & CTA & 46 & 1 (ORCR) \\
\hline 10 & $\mathrm{~F}$ & 61 & PHF (malunion) & 50 & 1 (osteotomy) \\
\hline 11 & $\mathrm{~F}$ & 61 & CTA & 34 & 0 \\
\hline 12 & M & 57 & CTA & 40 & 1 (ORCR) \\
\hline 13 & $\mathrm{~F}$ & 69 & CTA & 34 & 0 \\
\hline 14 & $\mathrm{~F}$ & 47 & CTA & 34 & 1 (ARCR) \\
\hline 15 & $\mathrm{~F}$ & 76 & PHF (nonunion) & 34 & 2 (ORIF, CR) \\
\hline 16 & $\mathrm{~F}$ & 61 & CTA & 32 & 1 (ORCR) \\
\hline 17 & $\mathrm{~F}$ & 69 & PHF (nonunion) & 31 & 1 (HA) \\
\hline 18 & $\mathrm{~F}$ & 65 & PHF (nonunion) & 29 & 1 (ORIF) \\
\hline 19 & M & 60 & CTA & 26 & 3 (ORCR) \\
\hline 20 & $\mathrm{~F}$ & 82 & CTA & 26 & 0 \\
\hline 21 & $\mathrm{~F}$ & 77 & CTA & 38 & 0 \\
\hline
\end{tabular}

$\mathrm{M}=$ male; $\mathrm{F}$ = female; $\mathrm{CTA}$ = cuff tear arthropathy; $\mathrm{PHF}=$ proximal humerus fracture; $\mathrm{ORCR}=$ open rotator cuff repair; $\mathrm{ARCR}=$ arthroscopic rotator cuff repair; ORIF = open reduction and internal fixation; $\mathrm{CR}=$ capsule release; HA $=$ hemiarthroplasty.

Patients had a mean followup of 44 months (range, 2681 months). No patients were lost to followup before 2 years, and all patients were seen in the last 5 years.

Pre- and postoperative ROM were compared; a goniometer was used, and measurements were done by the primary surgeons (LDH, JJPW). Active flexion and external rotation from the neutral and $90^{\circ}$ abducted positions were evaluated by physical examination. Degree of lag in external rotation was determined by comparing the passive and active ROM. All radiographs obtained during followup were examined for scapular notching by the surgeon. Any intraor postoperative complications were recorded. All data gathering was done retrospectively through chart review.

Pre- and postoperative pain visual analog scale (VAS) and the Single Assessment Numeric Evaluation (SANE), which is the percentage a patient would rate his or her shoulder with $100 \%$ being normal, were obtained [15]. Postoperative UCLA shoulder score and American Shoulder and Elbow Surgeons (ASES) shoulder score were analyzed.

All values are reported as mean with SD. Continuous variables were compared using the two-tailed MannWhitney test with significance accepted as $\mathrm{p}<0.05$. Microsoft Excel (Microsoft, Redmond, WA, USA) was used in the statistical analyses.

\section{Results}

Reverse shoulder arthroplasty with latissimus and teres major tendon transfers improved external rotation after surgery. Active external rotation with arm adducted increased from $6^{\circ} \pm 16^{\circ}$ preoperatively to $38^{\circ} \pm 14^{\circ}$ postoperatively (mean difference: $30^{\circ}$ [95\% confidence interval $\left.\left.\{\mathrm{CI}\}, 21^{\circ}-39^{\circ}\right], \mathrm{p}<0.001\right)$. The external rotation lag with arm adducted decreased from $40^{\circ} \pm 11^{\circ}$ preoperatively to $7^{\circ}$ $\pm 14^{\circ}$ postoperatively (mean difference: $-33^{\circ}[95 \% \mathrm{CI}$, $-43^{\circ}$ to $-22^{\circ}$ ], $\mathrm{p}<0.001$ ). Similarly, active external rotation with arm abducted increased from $19^{\circ} \pm 25^{\circ}$ preoperatively to $74^{\circ} \pm 22^{\circ}$ postoperatively (mean difference: $\left.44^{\circ}\left[95 \% \mathrm{CI}, 22^{\circ}-65^{\circ}\right], \mathrm{p}<0.001\right)$. The external rotation lag with the arm abducted decreased from $49^{\circ} \pm$ $28^{\circ}$ preoperatively to $5^{\circ} \pm 14^{\circ}$ postoperatively (mean difference: $-32^{\circ}\left[95 \% \mathrm{CI},-57^{\circ}\right.$ to $\left.\left.-7^{\circ}\right], \mathrm{p}<0.001\right)$. As expected, active forward flexion improved from $56^{\circ} \pm 36^{\circ}$ preoperatively to $112^{\circ} \pm 38^{\circ}$ postoperatively (mean difference: $64^{\circ}\left[95 \% \mathrm{CI}, 45^{\circ}-83^{\circ}\right.$ ], $\mathrm{p}<0.001$ ) (Table 2).

Improvements were seen in pain and outcome measures after reverse shoulder arthroplasty with latissimus dorsi and teres major transfers. The mean VAS pain score decreased from $8.4 \pm 2.3$ preoperatively to $1.7 \pm 2.1$ at the latest followup (mean difference: -6.9 [95\% CI, -8.7 to -5.2$]$, 


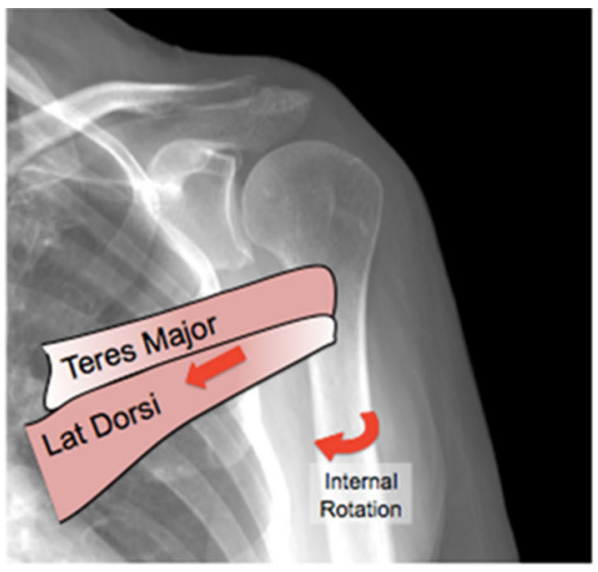

Before Tendon Transfer

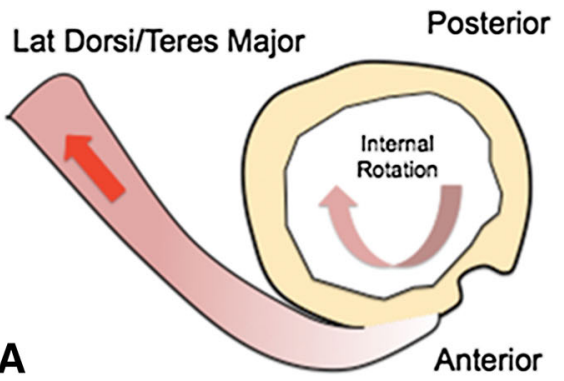

Fig. 1A-B Schematics are used to depict a latissimus dorsi ("lat dorsi") and teres major transfer in the setting of reverse shoulder arthroplasty. (A) Before the tendon transfer, the latissimus and teres major tendons attach on the anterior humerus and serve as internal

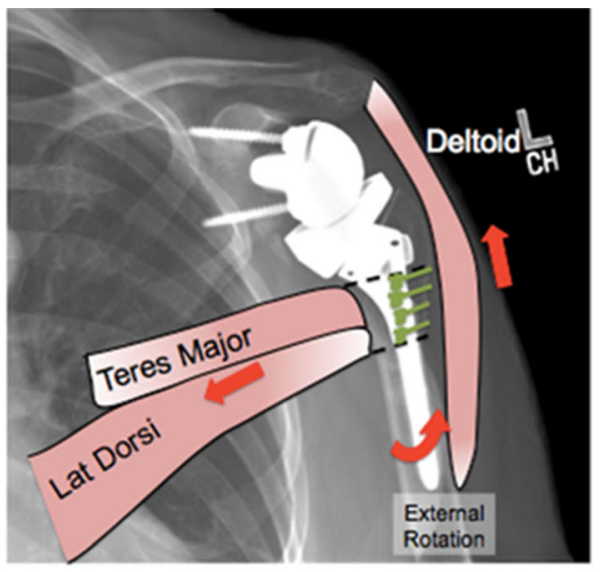

After Tendon Transfer

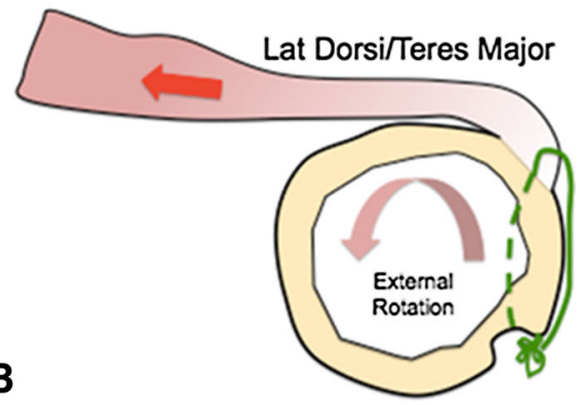

rotators. (B) After the reverse shoulder replacement and tendon transfers, the latissimus and teres major tendons are rerouted from the posterior humerus and attached to the lateral humerus. They then serve as external rotators.

Table 2. Clinical results

\begin{tabular}{|c|c|c|c|c|}
\hline ROM and outcome measurements & Preoperation & Postoperation & Mean difference $(95 \% \mathrm{CI})$ & $\mathrm{p}$ value \\
\hline Flexion & $56^{\circ} \pm 36^{\circ}$ & $120^{\circ} \pm 38^{\circ}$ & $64^{\circ}\left(45^{\circ}-83^{\circ}\right)$ & $<0.0001$ \\
\hline ER with arm adducted & $6^{\circ} \pm 16^{\circ}$ & $38^{\circ} \pm 14^{\circ}$ & $30^{\circ}\left(21^{\circ}-39^{\circ}\right)$ & $<0.0001$ \\
\hline ER lag with arm adducted & $40^{\circ} \pm 11^{\circ}$ & $7^{\circ} \pm 14^{\circ}$ & $-33^{\circ}\left(-43^{\circ}\right.$ to $\left.-22^{\circ}\right)$ & \\
\hline ER with arm abducted & $19^{\circ} \pm 25^{\circ}$ & $74^{\circ} \pm 22^{\circ}$ & $44^{\circ}\left(22^{\circ}-65^{\circ}\right)$ & $<0.0001$ \\
\hline ER lag with arm abducted & $49^{\circ} \pm 28^{\circ}$ & $5^{\circ} \pm 14^{\circ}$ & $-32^{\circ}\left(-57^{\circ}\right.$ to $\left.-7^{\circ}\right)$ & \\
\hline Pain VAS & $8.4 \pm 2.3$ & $1.6 \pm 2.0$ & $-6.9(-8.7$ to -5.2$)$ & $<0.0001$ \\
\hline UCLA & & $27 \pm 7$ & & \\
\hline ASES & & $74 \pm 23$ & & \\
\hline SANE & $28 \% \pm 21 \%$ & $80 \% \pm 24 \%$ & $46 \%(28 \%-64 \%)$ & $<0.0001$ \\
\hline
\end{tabular}

$\mathrm{CI}=$ confidence interval; ER = external rotation; VAS = visual analog score; ASES = American Shoulder and Elbow Surgeons; SANE = Single Assessment Numeric Evaluation.

$\mathrm{p}<0.001)$. The mean postoperative UCLA and ASES shoulder scores at the latest followup were $27 \pm 7$ and 74 \pm 23 , respectively. The SANE score improved from $28 \%$ $\pm 21 \%$ preoperatively to $80 \% \pm 24 \%$ postoperatively (mean difference: 46\% [95\% CI, 28\%-64\%], p < 0.001).

At the latest followup, radiographic results showed no humeral loosening; seven of 21 (33\%) developed scapula notching (five Grade 1 and two Grade 2), and no patients required revision based on scapula notching. Nine of 21
(43\%) patients experienced complications in our study (Table 3). They are classified as minor and major. There were three minor complications or complications that were beyond the surgeon's control. There was one intraoperative complication in which a patient had a humeral metaphyseal fracture that required cerclage wire fixation; the patient went on to an uneventful recovery (at 34 months her VAS was 1 , SANE $90 \%$, and ASES shoulder score 88). Two patients sustained postoperative falls leading to 
Table 3. Surgical complications

\begin{tabular}{ll}
\hline Complication type (major or minor) & $\begin{array}{l}\text { Number of } \\
\text { patients }\end{array}$ \\
\hline $\begin{array}{l}\text { Intraoperative metaphyseal fracture requiring cerclage } \\
\quad \text { wiring (minor) }\end{array}$ & 1 \\
$\begin{array}{l}\text { Fall and periprosthetic fractures requiring ORIF } \\
\text { (minor) }\end{array}$ & 1 \\
$\begin{array}{l}\text { Fall and periprosthetic fracture treated conservatively } \\
\quad \text { (minor) }\end{array}$ & 1 \\
$\begin{array}{l}\text { Prominent and painful baseplate fixation screw } \\
\quad \text { requiring surgery (major) }\end{array}$ & 1 \\
$\begin{array}{l}\text { Axillary nerve palsy treated conservatively (major) } \\
\text { Dislocation at } 2 \text { months requiring surgery (major) }\end{array}$ & 1 \\
$\begin{array}{l}\text { Acromion base fracture requiring surgery (major) } \\
\text { Deltoid detachment at } 1 \text { year requiring surgery }\end{array}$ & 1 \\
$\quad$ (major)
\end{tabular}

ORIF $=$ open reduction and internal fixation.

periprosthetic fractures, one of which required revision surgery. There were six major complications (six of 21 [29\%]), five of which required revision surgery. One patient's baseplate fixation screw was prominent at the tip as it penetrated the scapula spine; this required surgery to burr down the screw tip, and the patient was asymptomatic after the surgery. There was one patient with a postoperative axillary nerve palsy that was treated without surgery. One patient sustained anterior dislocation from a minor trauma at 2 months postoperatively; she underwent open reduction and humeral tray and polyethylene exchange. One patient experienced a traumatic acromion base fracture, which was treated using acromion open reduction and internal fixation with a tension band construct. Two patients had symptomatic deltoid detachment at 10 months and 1 year postoperatively; one of these patients had three prior open rotator cuff repairs, and the other had an arthroscopic cuff repair followed by revision open repair. Both underwent subsequent deltoid repair. Overall, three patients' latissimus and teres major transfers failed as defined by persistent external rotation lag of more than $30^{\circ}$ with the arm adducted and abducted.

\section{Discussion}

Because the infraspinatus and teres minor are the only external rotators of the shoulder, posterior rotator cuff deficiency can substantially compromise shoulder function. A rotator cuff tear leading to posterior cuff dysfunction or an injury to the suprascapular nerve and axillary nerve may affect the infraspinatus and the teres minor, respectively, leading to atrophy and dysfunction $[6,16]$. Since its development, reverse shoulder arthroplasty has shown to consistently decrease pain and restore active forward elevation and abduction [3, 10, 17], but without the infraspinatus and teres minor, loss of active external rotation persists [11]. Reverse shoulder arthroplasty with latissimus dorsi and teres major transfer has been reported to be a solution for this complex, and not uncommon, problem [2, 5]. What is available in the literature is limited with several small case series with short followup. Most reports do not have a consistent and well-defined surgical indication, and they have used different techniques. We therefore asked: (1) Do latissimus dorsi and teres major tendon transfers with reverse shoulder arthroplasty improve external rotation function? (2) Do patients experience less pain and have improved outcome scores after surgery? (3) What are the complications associated with reverse shoulder arthroplasty with latissimus dorsi/teres major transfer?

This study is limited by its followup duration (44 months; range, 26-81 months). It is possible if not likely that more of these reconstructions could fail in time, and we continue to follow these patients. We note, however, that followup of this group was complete; no patients were lost to followup, and so we are confident that we have captured all of the complications and reoperations that have happened as of the time of this report. The study's retrospective design is another potential limitation; retrospective studies often are limited by selection bias, which can inflate the apparent benefits of the treatment being studied. Certainly during the period of study not all patients with failed total shoulder arthroplasties and cuff deficiencies of this type were treated surgically, because not all were good surgical candidates. However, among those who were treated surgically and fulfilled the inclusion criteria during this period, all were managed with this approach, and so the influence of selection bias on our results should be limited for this reason. The concern for transfer bias is limited with patients from the earliest part of the study period having the longest followup, and all patients were seen in the last 5 years. There is no comparison group; such a control group is not possible and, indeed, is not ethical; in patients with loss of external rotation, if this transfer is not performed, it may result in a functional deficit for the patient. Indeed, no previous publications on this topic have had a control or performed randomization.

In a study by Boileau and colleagues [2], 17 patients (17 shoulders, mean age 71 years) who underwent reverse shoulder arthroplasty with latissimus dorsi and teres major transfer through a single deltopectoral approach were reported to have improvement in active forward flexion of $75^{\circ}$ and external rotation with the arm abducted of $34^{\circ}$, respectively. In another study, Boughebri and colleagues [5] reviewed 15 shoulders of 14 patients (mean age 67.5 years) who were treated with the same procedure; active forward flexion and external rotation improved postoperatively $61^{\circ}$ and $36^{\circ}$, respectively. Puskas and colleagues [9] published a series of 41 patients (41 shoulders) undergoing 
a similar procedure but through two incisions: one for tendon harvest and one for reverse shoulder arthroplasty and tendon transfer; they observed improvements of $62^{\circ}$ and $23^{\circ}$ in active forward flexion and external rotation, respectively. These previously reported improvements in forward flexion and external rotation are similar to our findings of $64^{\circ}$ and $30^{\circ}$, respectively.

Patients have improved pain and functional outcome after reverse shoulder arthroplasty with latissimus dorsi and teres major transfers.

Puskas et al. [9] reported improved Constant score from 45 to 89 after surgery, and Boughebri et al. [5] reported Constant score improved from $24 \%$ to 61 after surgery. We reported a significant decrease in VAS pain score from 8.4 to 1.7; our mean postoperative UCLA and ASES shoulder scores were 27 and 74, respectively. Patients have previously reported increases in subjective shoulder value with postoperative scores of $71 \%$ [2] and $75 \%$ [9], which is similar to our cohort's SANE score of $81 \%$.

Unlike previous studies, we observed a higher rate of complications. There were six major complications (six of 21 [29\%]), which was above the range of $12 \%$ (two of 17 ) to $22 \%$ (nine of 41) from other patients with short- to medium-term followup [2, 5, 9]. In reverse shoulder arthroplasty alone, there can be up to a $20 \%$ postoperative complication rate [18]. With 14 of 21 patients in our cohort having had at least one previous procedure, it is understandable that the complication rate is slightly higher. The types of complications were similar to those that occur with reverse arthroplasty alone, and none was directly attributed to the tendon transfers. For the two patients with deltoid detachment at 10 months and 1 year after surgery, both had prior open rotator cuff repairs, which likely contributed to the weakness of the deltoid attachment [14].

The increased complications did not appear to affect either the patients' clinical outcomes or their subjective assessment.

In patients with posterosuperior cuff deficiency, reverse shoulder arthroplasty combined with latissimus dorsi and teres major transfer through a single deltopectoral incision effectively increases active forward flexion and external rotation. Overall, patients experienced pain relief and improved function, but there can be a high rate of complications. We recommend reverse shoulder arthroplasty with latissimus dorsi and teres major transfer be limited to patients indicated to have a reverse shoulder arthroplasty who has profound loss of external rotation and a high grade of infraspinatus/teres minor fatty atrophy.

\section{References}

1. Boileau P, Chuinard C, Roussanne Y, Neyton L, Trojani C. Modified latissimus dorsi and teres major transfer through a single deltopectoral approach for external rotation deficit of the shoulder: as an isolated procedure or with a reverse arthroplasty. J Shoulder Elbow Surg. 2007;16:671-682.

2. Boileau P, Rumian A, Zumstein M. Reverse shoulder arthroplasty with modified L'Episcopo for combined loss of active elevation and external rotation. J Shoulder Elbow Surg. 2010;19:20-30.

3. Boileau P, Watkinson D, Hatzidakis AM, Balg F. Grammont reverse prosthesis: design, rationale, and biomechanics. $J$ Shoulder Elbow Surg. 2005;14(Suppl S):147-161S.

4. Boileau P, Watkinson D, Hatzidakis AM, Hovorka I. Neer Award 2005: The Grammont reverse shoulder prosthesis: results in cuff tear arthritis, fracture sequelae, and revision arthroplasty. $J$ Shoulder Elbow Surg. 2006;15:527-540.

5. Boughebri O, Kilinc A, Valenti P. Reverse shoulder arthroplasty combined with a latissimus dorsi and teres major transfer for a deficit of both active elevation and external rotation: results of 15 cases with a minimum of 2-year follow-up. Orthop Traumatol Surg Res. 2013;99;131-137.

6. Boykin RE, Friedman DJ, Higgins LD, Warner JJ. Suprascapular neuropathy. J Bone Joint Surg Am. 2010;92:2348-2364.

7. Gerber C, Pennington S, Lingenfelter E, Sukthankar A. Reverse Delta-III total shoulder replacement combined with latissimus dorsi transfer. A preliminary report. J Bone Joint Surg Am. 2007;89:940-947.

8. L'Episcopo JB. Tendon transplantation in obstetrical paralysis. Am J Surg. 1934;25:122-125.

9. Puskas G, Catanzaro S, Gerber C. Clinical outcome of reverse total shoulder arthroplasty combined with latissimus dorsi transfer for the treatment of chronic combined pseudoparesis of elevation and external rotation of the shoulder. J Shoulder Elbow Surg. 2014;23:49-57.

10. Ramirez MA, Ramirez J, Murthi AM. Reverse total shoulder arthroplasty for irreparable rotator cuff tears and cuff tear arthropathy. Clin Sports Med. 2012;31:749-759.

11. Simovitch RW, Helmy N, Zumstein MA, Gerber C. Impact of fatty infiltration of the teres minor muscle on the outcome of reverse total shoulder arthroplasty. J Bone Joint Surg Am. 2007;89:934-939.

12. Sirveaux F, Favard L, Oudet D, Huquet D, Walch G, Molé D. Grammont inverted total shoulder arthroplasty in the treatment of glenohumeral osteoarthritis with massive rupture of the cuff: results of a multicenter study of 80 shoulders. J Bone Joint Surg Br. 2004;86:388-395.

13. Werner CM, Steinmann PA, Gilbalt M, Gerber C. Treatment of painful pseudoparesis due to irreparable rotator cuff dysfunction with the Delta III reverse-ball-and-socket total shoulder prosthesis. J Bone Joint Surg Am. 2005;87:1476-1486.

14. Whatley AN, Fowler RL, Warner JJ, Higgins LD. Postoperative rupture of the anterolateral deltoid muscle following reverse total shoulder arthroplasty in patients who have undergone open rotator cuff repair. J Shoulder Elbow Surg. 2011;20:114-122.

15. Williams GN, Gangel TJ, Arciero RA, Uhorchak JM, Taylor DC. Comparison of the Single Assessment Numeric Evaluation method and two shoulder rating scales. Outcomes measures after shoulder surgery. Am J Sports Med. 1999;27:214-21.

16. Wilson L, Sundaram M, Piraino DW, Ilaslan H, Recht MP. Isolated teres minor atrophy: manifestation of quadrilateral space syndrome or traction injury to the axillary nerve? Orthopedics. 2006;29:447-450.

17. Young SW, Zhu M, Walker CG, Poon PC. Comparison of functional outcomes of reverse shoulder arthroplasty with those of hemiarthroplasty in the treatment of cuff-tear arthropathy: a matched-pair analysis. J Bone Joint Surg Am. 2013;95:910-915.

18. Zumstein MA, Pinedo M, Old J, Boileau P. Problems, complications, reoperations, and revisions in reverse total shoulder arthroplasty: a systematic review. J Shoulder Elbow Surg. 2011; 20:146-157. 